\title{
Gastrointestinal Diseases in Pregnancy; Diagnosis and Management
}

\author{
Neeraj Nagaich ${ }^{1 *}$, Radha Sharma ${ }^{2}$ and Niranjana Nair ${ }^{3}$ \\ ${ }^{1}$ Department of Gastroenterology FORTIS Escorts Hospital, India \\ ${ }^{2}$ Department of Pathology RUHS college of medical sciences, India \\ ${ }^{3}$ JSS college of pharmacy, India
}

*Corresponding author: Neeraj Nagaich, Fortis Escorts Hospital, India

Submission: 眥 September 26, 2018; Published: 眥January 22, 2019

\section{Introduction}

Gastrointestinal (GI) diseases are one of the most frequent complaints during pregnancy, susceptible mostly to nausea, vomiting, gastro esophageal reflux, constipation, and diarrhea at rates similar to or higher than the general population, possibly due to elevated levels of progesterone and/or prostaglandins. Although a vast majority of GI complaints are caused by normal pregnancyrelated changes, other pathologic conditions and causes should also be considered. When symptomatic remission cannot be achieved with nonpharmacologic therapy, pharmacologic treatment may be instituted, but the potential teratogenic side effects must be considered [1].

\section{Nausea and Vomiting}

Nausea and vomiting in pregnancy (NVP) affect nearly $80 \%$ of pregnant women. While $52 \%$ of women were found to have both nausea and vomiting, 28\% experienced nausea alone. The symptoms usually start within 4-7weeks after the last menstrual period and typically peak around 9weeks of gestation. Nausea and vomiting usually resolve by $14-20$ weeks of gestation in $60 \%$ and $97 \%$ of women respectively. However, some women experience reemergence of symptoms in the last few weeks before delivery [2]. Although the term morning sickness is commonly used to describe nausea and vomiting of pregnancy, the timing, severity, and duration of symptoms vary widely. Approximately $80 \%$ of women report that their symptoms last all day, whereas only $1.8 \%$ report symptoms that occur solely in the morning [3].

\section{Risk factors}

Data from the Collaborative Perinatal Project found NVP to be more common in younger women, primigravidas, women with less than 12 years of education, nonsmokers, and obese women [4]. Other risk factors for NVP include a personal history of motion sickness and history of migraine headaches and nausea when taking estrogen-containing oral contraceptives. Location of the corpus luteum may also serve as risk factor for NVP (more nausea and vomiting when the corpus luteum is present in the right ovary) [5].

\section{Pathophysiology}

The exact cause of nausea and vomiting during pregnancy is not fully understood. Metabolic and hormonal Factors are levels of estrogen and progesterone (by reducing intestinal motility and gastric emptying via the nitric oxide pathway), Human Chorionic Gonadotropin (hcg) (based largely on the temporal relationship between the peak of NVP and the peak of hcg production, both of which occur between 12 and 14 weeks gestation), placental prostaglandins (by regulating gastric slow-wave frequency and peristalsis), serotonin levels, thyroid dysfunction (due to the cross-reactivity between hcg and the TSH receptor) and increased leptin levels. Other potential etiologies include immune system dysregulation, Helicobacter pylori infection, and gastrointestinal dysmotility (alterations in lower esophageal sphincter tone) and psychosocial Factors (unplanned or undesired pregnancies, neurotic tendencies, hysteria, rejection of femininity, and rejection of pregnancy, depression and psychological stress related to poverty and marital conflicts) [1-3,5].

\section{Hyperemesis Gravidarum}

About 1\% of women develop hyperemesis gravidarum, which may result in adverse outcomes for the mother and fetus [3]. The International Statistical Classification of Disease and Related Health Problems, Tenth Revision, defines hyperemesis gravidarum (HG) as persistent and excessive vomiting starting before the end of the $22^{\text {nd }}$ week of gestation and associated with metabolic disturbances such as carbohydrate depletion, dehydration, or electrolyte imbalance [6], weight loss, ketonuria, electrolyte disturbances, dehydration, and/or hospitalization. It is associated with a higher risk of negative pregnancy outcomes: preterm birth, low birth weight, and small-for gestational-age infants. Risk factors for HG include female gender infant, young maternal age, non-European heritage [1], multiple gestation, molar pregnancy, fetal abnormalities, and women with medical conditions such as hyperthyroid disorders, preexisting diabetes, obesity and asthma [2]. Body mass index, smoking, and socioeconomic status are not risk factors for HG [1]. 


\section{Pathophysiology}

The cause of HG is understood poorly. There is a growing body of literature suggests 3 pathoetiologies for HG: placental growth and function as reflected by free human chorionic gonadotropin (hCG), maternal endocrine function (Table 1), and preexisting gastrointestinal disease. In addition, psychopathologic condition could play a role in the cause of HG like stress, depression, and anxiety. Infection with Helicobacter pylori may also play a role in the development of HG in some women. The cause for the increased incidence of $\mathrm{H}$ pylori in patients with $\mathrm{HG}$ is unclear and no causal relationship between $\mathrm{HG}$ and $\mathrm{H}$ pylori has been established. HG therefore seems to be a heterogeneous illness [1,6-9] (Table 1).
Table 1: Sex hormone and endocrine factors associated with hyperemesis gravidarum [1].

\begin{tabular}{|c|c|}
\hline Factor & Alternation \\
\hline Sex hormones & \\
\hline Estrogens & Increased \\
\hline Progesterone & Increased \\
\hline Endocrine hormones & \\
\hline Thyroid-stimulating hormone and thyroxine & Overactivity \\
\hline Hypothalamic-pituitary-adrenal axis & Overactivity \\
\hline Human growth hormone & Abnormal levels \\
\hline Prolactin & Abnormal levels \\
\hline
\end{tabular}

\section{Differential diagnosis}

Table 2: Differential diagnosis for nausea and vomiting in pregnancy [5].

\begin{tabular}{|c|c|c|c|c|}
\hline GI Disorders & Genitourinary Disorders & Metabolic Disorders & Neurologic Disorders & Pregnancy-Related Disorders \\
\hline Gastroenteritis & Nephrolithiasis & Hyperthyroidism & Migraines & Preeclampsia/HELLP \\
\hline GERD & Pyelonephritis & Addison's disease & CNS tumors & Acute fatty liver of pregnancy \\
\hline $\begin{array}{c}\text { Peptic ulcer disease } \\
\text { Intestinal obstruc- } \\
\text { tion }\end{array}$ & Ovarian torsion & Diabetic complications & Pseudotumor cerebri & \\
\hline $\begin{array}{c}\text { Pancreatitis } \\
\text { Appendicitis }\end{array}$ & & & Vestibular abnormalities & \\
\hline Hepatitis & & & & \\
\hline Biliary disorders & & & & \\
\hline
\end{tabular}

Diagnostic and laboratory tests (Table 2): Other than a pregnancy test, no specific laboratory studies are recommended for the diagnosis of NVP. Leukocytosis should not be seen in NVP and may point to an infectious or inflammatory cause such as cholecystitis, urinary tract infections, or pancreatitis. Elevations in the aminotransferases could indicate chronic hepatitis but may also be the result of repetitive vomiting. An abnormal TSH could indicate hypothyroidism or hyperthyroidism, both of which can cause nausea and vomiting. Elevation in serum glucose could indicate diabetes and may produce nausea and vomiting by decreasing antral contractility and precipitating gastric dysrhythmias. Upper endoscopy can be performed safely in pregnancy and can be considered to rule out gastritis and peptic ulcer disease as causes of nausea and vomiting in pregnancy [5].

It is important to emphasize that early assessment of nausea and vomiting in pregnancy is essential to prevent delay in diagnosis and management of HG. A pregnancy-unique quantification of emesis and nausea (PUQE) score, calculated using the number of hours of nausea per day, number of episodes of emesis per day, and number of episodes of retching per day, can be used to track the severity of symptoms $[5,6]$.
Notably, diagnostic biomarkers for HG have produced inconsistent results. Although ketonuria is often assessed as part of a clinical examination, the robustness of ketonuria as a diagnostic marker for HG remains unclear. Future investigations examining ketonuria levels in the diagnosis and severity of $\mathrm{HG}$ are warranted. Lymphocytes were typically higher in women presenting with HG, although the association between HG and hCG and thyroid hormones, leptin, estradiol, progesterone, and white blood count were less reliable. H. pylori serology may be of diagnostic benefit [1].

Management: After other physiologic causes for nausea and vomiting have been eliminated, nonpharmacologic treatment options should be instituted [1].

\section{A. Nonpharmacologic therapies}

a) Dietary modifications: Traditional first-line therapy for nausea and vomiting of pregnancy and for hyperemesis gravidarum includes dietary modifications. Avoid large meals and instead eat several small meals throughout the day, also avoid consumption of low-fat, low-fiber, bland foods (e.g., breads, crackers, cereals, eggs, lean meat, peanut butter, fruits, fresh vegetables) that may delay gastric emptying. High-protein liquid beverages have been shown 
to reduce gastric dysrhythmias and nausea in the first trimester associated with NVP. To prevent dehydration, daily consumption of $1 \mathrm{~L}$ to $1.5 \mathrm{~L}$, either sports drinks or broth containing salt, glucose, and potassium might be helpful $[3,5]$.

b) Emotional support: Emotional support, adequate rest, supportive psychotherapy, behavioral therapy, and hypnotherapy may be beneficial to women with severe symptoms and/or those in whom personality characteristics and/or marital or family conflict play a role [5].

c) Acupressure/acupuncture: Studies have shown that acupressure of the Neiguan point- P6 (three finger breaths above the wrist on volar surface) is helpful in controlling nausea in early pregnancy. According to the principle of Chi, application of pressure to this point blocks abnormal energy slowly and relieves symptoms related to the pressure point. Pressure may be placed manually or with elastic bands on the inside of the wrist. In addition, the Relief Band, a battery-operated electrical nerve stimulator worn on the wrist, has been approved by FDA and can also be used to stimulate the P6 site. The evidence for acupressure is largely positive; however, they are inconclusive in validating the role of acupressure on symptom relief. Since this intervention is free from any adverse effects it ought to be recommended to patients for symptom relief $[2,5]$.

d) Ginger: Zingiber officinale, the root of ginger is believed to help improve NVP by stimulating GIT motility and stimulating the flow of saliva, bile, gastric secretions and block the cholinergic $\mathrm{M}$ receptors as well as the serotonin receptor, after daily consumption of $1 \mathrm{~g}$ of ginger. In addition, its extract has been found to inhibit the growth of some strains of $\mathrm{H}$ pylori. Theoretical risks for bleeding exist, as ginger inhibits thromboxane synthetase and may inhibit platelet function. Thus, in women with an increased bleeding risk and concomitant use of anticoagulants with ginger is not advised $[2,5]$.

\section{B. Pharmacologic treatment}

Due to the concern for teratogenic effects, judicious use of all pharmacologic therapy in pregnant women is indicated. The Food and Drug Administration (FDA) has classified many supplements as well as over-the-counter and prescription medications in 1 of 5 pregnancy categories $(\mathrm{A}, \mathrm{B}, \mathrm{C}, \mathrm{D}$, or $\mathrm{X}$ ) based on the risk of developmental and reproductive adverse effects compared with the potential benefit (Table 3).

Table 3: Definition of food and drug agency pregnancy classifications [1].

\begin{tabular}{|c|c|}
\hline Class & Definition \\
\hline A & Appropriately designed studies in pregnant women have not demonstrated fetal risk. \\
\hline B & No fetal risk in animal reproduction studies; no well-controlled studies in pregnant women \\
\hline & Adverse effect in animal studies, but studies in pregnant women failed to demonstrate fetal risk \\
\hline C & Animal reproduction studies show adverse fetal effect but no well controlled studies in humans. \\
\hline & No animal reproduction studies and well-controlled studies in humans \\
\hline D & Evidence of human fetal risk in investigational or marketing experience or studies in humans \\
\hline $\mathrm{X}$ & Animals or humans studies have demonstrated fetal abnormalities. \\
\hline & Evidence of fetal risk based on investigational and/or marketing experience \\
\hline
\end{tabular}

Regarding the pharmacologic treatment of nausea and vomiting, antiemetics (most commonly used agents phenothiazines, histamine receptor blockers, and dopamine antagonists) should be used cautiously in pregnancy and should not be used before 12 to 14 weeks of gestation. Ondansetron, a 5-HT3 antagonist, is particularly useful in the treatment of chemotherapy-induced nausea and vomiting. Steroids, which are thought to relieve nausea via a

Table 4: Pharmacologic treatments for nausea and vomiting of pregnancy [3]

\begin{tabular}{|c|c|c|c|c|}
\hline Agent & Dosage & Adverse effects & Fotes category \\
\hline \multicolumn{3}{|c|}{ Primary therapies } \\
\hline Vitamin B6 & $10-25 \mathrm{mg}$ every 8 hours & $\begin{array}{c}\text { Paresthesias, headache, } \\
\text { fatigue }\end{array}$ & First line therapy A \\
\hline
\end{tabular}




\begin{tabular}{|c|c|c|c|c|}
\hline Doxylamine & $\begin{array}{l}12.5 \text { to } 25 \mathrm{mg} \text { every eight } \\
\text { hours }\end{array}$ & Drowsiness & $\begin{array}{c}\text { May use lower dose in the morning and at midday, and } \\
\text { larger dose at night }\end{array}$ & $\mathrm{B}$ \\
\hline \multicolumn{5}{|c|}{ Secondary therapies } \\
\hline $\begin{array}{l}\text { Dimenhydri- } \\
\text { nate }\end{array}$ & $\begin{array}{l}50 \text { to } 100 \mathrm{mg} \text { every four } \\
\text { to six hours }\end{array}$ & $\begin{array}{l}\text { Drowsiness, dizziness, } \\
\text { headache, fatigue }\end{array}$ & ------ & $\mathrm{B}$ \\
\hline $\begin{array}{l}\text { Diphenhydr- } \\
\text { amine }\end{array}$ & $\begin{array}{l}25 \text { to } 50 \text { mg every eight } \\
\text { hours }\end{array}$ & $\begin{array}{l}\text { Drowsiness, dizziness, } \\
\text { headache, fatigue }\end{array}$ & -------- & $\mathrm{B}$ \\
\hline Hydroxyzine & $\begin{array}{l}50 \mathrm{mg} \text { every four to six } \\
\text { hours }\end{array}$ & $\begin{array}{l}\text { Drowsiness, dizziness, } \\
\text { headache, fatigue }\end{array}$ & ------ & \\
\hline Meclizine & $25 \mathrm{mg}$ every six hours & $\begin{array}{l}\text { Drowsiness, dizziness, } \\
\text { headache, fatigue }\end{array}$ & ------- & $\mathrm{B}$ \\
\hline $\begin{array}{l}\text { Metoclopra- } \\
\text { mide }\end{array}$ & $10 \mathrm{mg}$ every six hours & Tardive dyskinesia & Avoid high dosages or treatment for longer than 12 weeks & $\mathrm{B}$ \\
\hline Ondansetron & 4 to $8 \mathrm{mg}$ every six hours & $\begin{array}{l}\text { Headache, diarrhea, con- } \\
\text { stipation, fatigue }\end{array}$ & Available as oral disintegrating tablet & $\mathrm{C}$ \\
\hline Promethazine & $\begin{array}{l}12.5 \text { to } 25 \mathrm{mg} \text { every four } \\
\text { to six hours }\end{array}$ & $\begin{array}{l}\text { Sedation, extrapyramidal } \\
\text { symptoms }\end{array}$ & $\begin{array}{l}\text { Avoid intravenous administration (associated with tissue } \\
\text { damage); oral, rectal, or intramuscular administration is } \\
\text { safer; can be compounded as transdermal gel }\end{array}$ & $\mathrm{C}$ \\
\hline \multicolumn{5}{|c|}{ Therapies for refractory nausea and vomiting } \\
\hline Droperidol & $\begin{array}{l}1.25 \text { to } 2.5 \mathrm{mg} \text { intramus- } \\
\text { cularly or intravenously }\end{array}$ & $\begin{array}{l}\text { Torsades de pointes, pro- } \\
\text { longed QT interval }\end{array}$ & ---- & $\mathrm{C}$ \\
\hline $\begin{array}{l}\text { Methylprednis- } \\
\text { olone }\end{array}$ & $\begin{array}{l}\text { 16mg every eight hours } \\
\text { for three days, then } \\
\text { taper over two weeks }\end{array}$ & ----- & $\begin{array}{l}\text { Increased risk of cleft lip if used before } 10 \text { weeks' gesta- } \\
\text { tion; data on effectiveness are mixed; may be given orally } \\
\text { or intravenously }\end{array}$ & $\mathrm{C}$ \\
\hline $\begin{array}{l}\text { Prochlorper- } \\
\text { azine }\end{array}$ & $\begin{array}{l}5 \text { to } 10 \text { mg every six } \\
\text { hours }\end{array}$ & $\begin{array}{l}\text { Sedation, extrapyramidal } \\
\text { symptoms, hypotension }\end{array}$ & Available as buccal tablet & \\
\hline Scopolamine & $\begin{array}{l}\text { 1-mg patch behind ear } \\
\text { every three days }\end{array}$ & $\begin{array}{l}\text { Drowsiness, dry mouth, } \\
\text { urinary retention }\end{array}$ & $\begin{array}{c}\text { May cause limb and trunk deformities if used in first } \\
\text { trimester }\end{array}$ & \\
\hline $\begin{array}{l}\text { Trimethoben- } \\
\text { zamide }\end{array}$ & $\begin{array}{l}300 \mathrm{mg} \text { every six to eight } \\
\text { hours }\end{array}$ & $\begin{array}{l}\text { Sedation, anticholinergic } \\
\text { effects }\end{array}$ & ---- & \\
\hline
\end{tabular}


a) Intravenous fluids: Fluid replacement is safe and effective in restoring volume and electrolytes in women who have HG and are unable to tolerate oral intake. Lactated Ringer solution or normal saline or Hartmann solution (potassium chloride can be added as needed) is acceptable. Because of the risk of Wernicke encephalopathy, intravenous thiamine should be added if dextrosecontaining fluids are administered, if vomiting has lasted longer than three weeks, or if fluid replacement lasts longer than three days $[3,9]$.

b) Enteral and parenteral nutrition: Patients who have refractory nausea and vomiting may require hospitalization. In these patients, enteral tube feeding in addition to routine intravenous fluids may be helpful. If patients do not respond to this therapy, parenteral nutrition may be necessary. Administration of parenteral nutrition is associated with significant risk during pregnancy, including a $25 \%$ risk of line sepsis, as well as steatohepatitis if lipid emulsion is used. Therefore, it should be reserved for extreme cases that have been refractory to enteral nutrition [3] (Table 3). Data from Administration FaD. Content and format of labeling for human prescription drug and biological products; requirements for pregnancy and lactation labeling. Fed Regist vol.2008:30831-68. Codified at 30821 CFR 30201.

\section{Gastroesophageal Reflux Disease (GERD)}

GERD is a disease characterized by abnormal reflux of gastric contents into the esophagus. Approximately 45-80 \% of women will experience GERD symptoms. In women with a diagnosis of GERD prior to pregnancy, symptoms can worsen as their pregnancy progresses. The clinical features of GERD are similar in pregnancy as in the nonpregnant general population. Symptoms include burning, acid taste, regurgitation, and provocation after meals and when lying supine [10]. When present, GERD persists during the entire pregnancy and usually resolves after delivery [11]. Although symptoms may be severe, erosive esophagitis, strictures, and bleeding are rare complications. Risk factors for heartburn in pregnancy include multiparty, preexisting heartburn, and gestational age. Studies have not shown an association between race, prepregnancy body mass index, or weight gain during pregnancy and the development of GERD [1].

\section{Pathophysiology}

The pathogenesis of GERD during pregnancy is likely multifactorial (Both mechanical and hormonal factors) and related to a series of functional and structural changes. Possible factors that contribute to GERD symptoms during pregnancy include reduced lower esophageal sphincter (LES) pressure (presence of estrogen, progesterone) and impaired LES contractility in response to pharmacologic stimuli. Hormonally induced alterations in gastric motility have also been implicated. Hormones are thought to effect the function of the enteric nervous system and musculature that leads to a decrease in GI motility, which may promote GERD. Whereas increased abdominal pressure from the enlarged gravid uterus is less likely to explain symptoms because heartburn is typically reported early in pregnancy $[1,10]$.

\section{Management}

The diagnosis of GERD can be made based on symptoms and more invasive testing during pregnancy is typically not necessary. Barium radiographs are avoided during pregnancy due to concern for fetal radiation exposure. Esophageal manometry and pH studies are safe but are rarely necessary during pregnancy. Signs and symptoms of dysphagia, odynophagia, and significant or continued gastrointestinal bleeding are considered to be indications for esophagogastroduodenoscopy (EGD), during pregnancy by the American Society for Gastrointestinal Endoscopy (ASGE) $[1,10]$.

Nonpharmacologic therapies: For mild symptoms, lifestyle and dietary modifications are recommended. Conservative measures include avoiding eating and drinking late at night (within $4 \mathrm{~h}$ of bedtime), by elevating the head of the bed by $10-15 \mathrm{~cm}$ and lying on the left side, nocturnal reflux symptoms can sometimes be alleviated. If symptoms persist, medication may be necessary. The patient should be advised to avoid fatty foods, citrus juices, caffeine, chocolate, mint, NSAIDs, alcohol, and smoking. Food diary to determine foods that trigger symptoms, avoiding excessive weight gain in pregnancy, increase between-meals water consumption, Apple cider vinegar, Aloe juice: $1 / 2$ cup before meals, Ginger root: 2-3 slices steeped in hot water; drink 8 oz before meals are other non-pharmacological treatments. When symptoms are refractory to these interventions then a trial of medical therapy is appropriate [11-13].

Pharmacologic treatment: Unfortunately, many of the medications used to treat GERD in pregnancy have not been tested in randomized clinical trials in this patient population [1]. For the pregnant patient with mild to moderate GERD symptoms, initial therapy can begin with an antacid (calcium- and magnesium-based antacids) in normal therapeutic doses. Bicarbonate-containing agents, such as sodium bicarbonate, should be avoided as they can precipitate metabolic alkalosis and fluid overload in the mother and fetus. Aluminum antacids is the potential developmental retardation described when women take high doses of this medication.

In patients with more-severe symptoms who have not responded to antacid therapy, histamine- 2 receptor antagonists are often effective in controlling symptoms of reflux and promoting ulcer healing. Examples are cimetidine, ranitidine, famotidine and nizatidine, which are all classified as category B drugs during pregnancy but, omeprazole category C. All agents that decrease gastric acidity should be used with caution, as they can lower iron absorption. Altogether, PPIs are nowadays considered to be safe in pregnant women and are usually reserved for GERD complications or for women who did not respond to previous treatments [10-12].

Sucralfate, one gram 3 times daily, has been shown more effective in inducing symptomatic remission than lifestyle management alone. No adverse maternal or fetal events were reported. Metoclopramide, a promotility agent, is primarily used in the treatment of pregnancy associated nausea and vomiting. 
Nonetheless, it can also improve the symptoms of GERD by promoting gastric emptying, improving acid clearance, and increasing LES pressure. No congenital malformations or fetal toxicities have been associated with its use but, long-term use can cause neurologic complications, such as dystonia and akathisia [10].

\section{Constipation}

Constipation is the second most common GI complaint in pregnancy. Typically, the symptoms of constipation are most prevalent in the first and second trimesters, 35\% and 39\% of women, respectively and decrease in the third. For unknown reasons, subsequent pregnancies have a higher risk for constipation $[1,11]$. Anderson et al. [14] reported that $38 \%$ and $20 \%$ of women experienced constipation in the second and third trimesters respectively. More recent data from Marshall et al. reported that $35 \%$ of women suffered from constipation during pregnancy $[13,14]$. The signs and symptoms included in the ROME III criteria are frequency of stools ( $<3$ per week), straining, lumpy or hard stools, incomplete evacuation, sensation of anal obstructions, and manual maneuvers to aid with defecation. Straining, hard or lumpy stools and incomplete evacuation have been found to be the most common complaints in pregnancy, whereas infrequent defecation is not as common [13]. Risk factors for constipation include a sedentary lifestyle, bed rest, low fiber intake, and inadequate fluid intake. Medications, such as iron, may also contribute to constipation [11].

\section{Pathophysiology}

The cause of constipation in pregnancy is often multifactorial because constipation is more prevalent in early pregnancy; sex hormones are thought to be involved. During normal pregnancy, serum concentrations of progesterone are elevated and progressively rise, leading to hypomotility of the small and large bowel. Additionally, the formation of hard, scybalous stool may be caused by aldosterone mediated increased colonic water absorption. Elevated levels of progesterone cause increased concentrations of aldosterone. Additionally, decreased colonic smooth muscle contractility prolongs colonic transit time, which could also contribute to stool dehydration. Iron intake during pregnancy can worsen constipation complaints. Finally, mechanical factors can also contribute in late pregnancy. The combined movements of the intestinal tract and uterus have been found to impede the onward movement of solid feces, obstruct defecations and initiate constipation [1,11].

\section{Diagnostic and laboratory tests}

History as always is an important part of the clinical evaluation. The physical examination may provide little or no information in cases of simple constipation. Clinical evidence of conditions such as hypothyroidism or diabetes mellitus should be sought. Examination of the perineum and anal canal is of importance. Lab investigations are aimed at excluding treatable disorders such as hypothyroidism or hypercalcaemia. A full blood count, thyroid-stimulating hormone (TSH), serum calcium and glucose should be performed in all patients presenting with constipation. Endoscopy is rarely indicated in the pregnant patient presenting with constipation unless there is compelling evidence for a need to exclude conditions such as colorectal neoplasia [15].

\section{Management}

Non-pharmacological management of constipation includes adequate water intake and a high-fiber diet. Food volume may exacerbate the symptoms, especially in the second trimester, and fractionized meals may be an alternative [11]. Strenuous exercise may worsen constipation, but light physical activity can promote normal bowel function. Dietary adjustments include increasing fluid intake ( $>8$ glasses per day) and fiber consumption $(>20-35 \mathrm{~g}$ per day). Intermittent iron supplementation can be as effective as daily dosing in the treatment of anemia but may reduce constipation [1]. When lifestyle and diet modifications do not provide adequate symptomatic relief, bulk-forming agents are usually considered. Because they are not systemically absorbed, these medications are thought to be safe in pregnancy and may be used for long durations.

A. Bulk-forming laxatives: Dietary modification, through increased fiber intake and ensuring adequate liquid consumption, should be the first-line therapy for constipation in pregnancy. psyllium, methylcellulose, guar, calcium polycarbophil, pectin, and/ or flax seed (ground or whole) to $25-40 \mathrm{~g}$ /day of fiber is safe and often effective

B. Hyperosmolar laxatives: Polyethylene glycol (PEG) and the nonabsorbable sugars such as lactulose, sorbitol, and glycerin are osmotic laxatives that stimulate the accumulation of fluid in the GI lumen. Studies have shown that PEG (FDA class C) can accelerate colorectal transit, increase the frequency of bowel movements, and improve defecation in patients with constipation refractory to dietary fiber. There use is often limited by bloating and cramping. Saline hyperosmotic laxatives, such as phosphorus salts and magnesium containing laxatives can result in sodium retention in the mother and are best avoided.

C. Lubricant laxatives: Use of the lubricant laxative mineral oil should be limited to short periods, because of the possibility of malabsorption of vitamins and nutrients, which could lead to hemorrhage and neonatal hypoprothrombinemia. Due to concern for premature uterine contractions induced by castor oil they are not recommended. Oral administration should be avoided in patients with impaired swallowing, because of the risk of developing aspiration pneumonia.

D. Stimulant laxatives: It is reasonable to consider use of stimulant laxatives, if osmotic and bulk laxatives fail to relieve the constipation. The stimulant laxatives senna and bisacodyl seem to be safe in pregnancy, also minimally systemically absorbed but FDA class $\mathrm{C}$ and have not been reported to be teratogenic. Oral bisacodyl often produces cramping, which limits its use. Stimulant laxatives such as castor oil can induce uterine contractions in the mother and should be avoided. Lubiprostone, a fatty acid derivative 
prostaglandin E1, causes increased ion and fluid secretion inside the GI lumen $[1,12]$.

\section{Diarrhea}

Compared with other GI disorders in pregnancy, there is significantly less literature on diarrhea in pregnancy [1]. Levy and associates in 1970s reported more frequent bowel movements in $34 \%$ of women during pregnancy [16].

\section{Etiology of acute diarrhea}

The causes of diarrhea in pregnancy are similar to that in the nonpregnant Patient. The most common causes of diarrhea in pregnancy are infectious agents, such as bacterias (like Salmonella species, Shigella species, Campylobacter species, Escherichia coli), protozoans (Giardia, Cryptosporidium, Entamoeba histolytica), and viruses (most commonly calciviruses, rotavirus, and adenovirus). Food poisoning may also cause diarrhea. Medications, malabsorption, and maldigestion are occasionally found to cause diarrhea $[13,16]$.

\section{Pathophysiology}

Gastrointestinal tract motility has not been studied in pregnant women complaining of diarrhea. In theory, increased prostaglandins might induce diarrhea by stimulating smooth muscle contractions and increasing the gut propulsive force and by causing gut mucosal secretion of water and electrolytes. These effects have been reported for misoprostol, an exogenous prostaglandin [16].

\section{Evaluation}

Evaluation of acute diarrhea is warranted if any of the following symptoms are present: persistent diarrhea, weight loss, malnutrition, fever, or signs of volume depletion. Stool assay should be done for an infectious cause and also it is necessary to rule out noninfectious. If necessary, a flexible sigmoidoscopy may be performed to evaluate the colonic mucosa, which is safe in pregnancy and is not associated with the induction of labor or congenital malformations [1].

\section{Management}

Uncomplicated cases can be treated with supportive therapy in the form of correction of fluid losses (water, clear juices, or broth) and electrolyte abnormalities (eg. Pedialyte) and dietary changes (Foods high in fat and lactose should be avoided. Bland foods and foods low in fiber may be beneficial. Restrictive diet- BRAT (bananas, rice, applesauce, and toast) has been shown to decrease the nausea associated with diarrheal episodes, although there is no evidence that restricted diets decrease the frequency of diarrhea stools). If symptoms remain persistent, limited use of antidiarrheal agents can be considered $[12,13]$.

Loperamide is probably safe, although it has been inadequately studied, and its routine use is not recommended [16]. Once an FDA class B medication, loperamide was reclassified as category $\mathrm{C}$ due to follow-up studies that showed an increased risk of hypospadias, caesarean section, placenta previa, and large-for-gestational-age infants in women who reported using loperamide in early pregnancy. Other common antidiarrheal agents, including diphenoxylate with atropine and bismuth subsalicylate (potential for salicylate absorption, which is associated with associated with prolonged labor and gestation, increased perinatal mortality, decreased birth weight, and neonatal hemorrhage), are contraindicated in pregnancy. Many of the antibiotics used to treat infectious diarrhea are contraindicated throughout or during specific periods of gestation; these include the quinolones, tetracyclines, sulfa preparations, and metronidazole. In severe cases antibiotics with a safer pregnancy profile, such as erythromycin and ampicillin can be given [1].

\section{References}

1. Body C, Christie JA (2016) Gastrointestinal diseases in pregnancy. Gastroenterol Clin North Am 45(2): 267-283.

2. Ross JA (2017) The McGovern Medical School Journal of Medicine. Editor-in-Chief 1(2).

3. Herrell HE (2014) Nausea and vomiting of pregnancy. Am Fam Physician 89(12): 965-970.

4. Klebanoff MA, Koslowe PA, Kaslow R, Rhoads GG (1985) Epidemiology of vomiting in early pregnancy. Obstet Gynecol 66(5): 612-616.

5. Lee NM, Saha S (2011) Nausea and vomiting of pregnancy. Gastroenterol Clin North Am 40(2): 309-334.

6. McCarthy FP, Lutomski JE, Greene RA (2014) Hyperemesis gravidarum: Current perspectives. Int J Womens Health 6(1): 719-725.

7. Frias C, Sousa M, Lourenço I, Martins D, Torres J (2018) Gastrointestinal diseases during pregnancy :what does the gastroenterologist need to know? Ann Gastroenterol 31(4): 385-394.

8. Verberg MF, Gillott DJ, Al-Fardan N, Grudzinskas JG (2005) Hyperemesis gravidarum, a literature review. Hum Reprod Update 11(5): 527-539.

9. Niemeijer MN, Grooten IJ, Vos N, Bais JMJ, Van Der Post JA, et al. (2014) Diagnostic markers for hyperemesis gravidarum: A systematic review and metaanalysis. Am J Obstet Gynecol 211(2): 150.

10. Castillo MJ, Phillippi JC (2015) Hyperemesis gravidarum. J Perinat Neonatal Nurs 29(1): 12-22.

11. Akbari M, Wolf JL, Adalimumab ADA (2017) Medical Problems During Pregnancy.

12. Thukral C, Wolf JL (2006) Therapy insight: Drugs for gastrointestinal disorders in pregnant women. Nat Clin Pract Gastroenterol Hepatol 3(5): 256-266.

13. Zielinski R, Searing K, Deibel M (2015) Gastrointestinal distress in pregnancy: Prevalence, assessment, and treatment of 5 common minor discomforts. J Perinat Neonatal Nurs 29(1): 23-31.

14. Anderson AS (1986) Dietary factors in the aetiology and treatment of constipation during pregnancy. Br J Obstet Gynaecol 93(3): 245-249.

15. Cullen G, O’Donoghue D (2007) Constipation and pregnancy. Best Pract Res Clin Gastroenterol 21(5): 807-818.

16. Bonapace J, Fisher RS (1998) Constipation and diarrhea in pregnancy. Gastroenterol Clin North Am 27(1): 197-211. 
Creative Commons Attribution 4.0 International License

For possible submissions Click Here

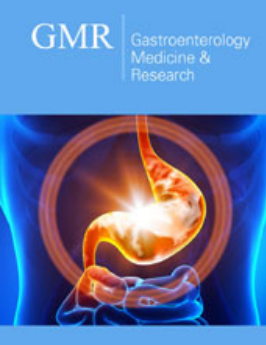

Gastroenterology Medicine \& Research

\section{Benefits of Publishing with us}

- High-level peer review and editorial services

- Freely accessible online immediately upon publication

- Authors retain the copyright to their work

- Licensing it under a Creative Commons license

- Visibility through different online platforms 